Publ. RIMS, Kyoto Univ.

13 (1977), 277-284

\title{
On an $L^{p}$-Estimate of Resolvents of Markov Processes
}

\author{
By \\ Masatoshi FuKUshIMA*
}

\section{$\S 1$. Introduction}

Let $(X, \mathscr{B}, m)$ be a measure space and $L^{p}=L^{p}(X ; m)$ be the real $L^{p}$-space with norm \|\|$_{p} . \quad L^{2}$-inner product is denoted by $(, \quad)$. The notation $\mathcal{E}$ will always means a bilinear form defined on $\mathscr{D}[\mathcal{E}] \times \mathscr{D}[\mathcal{E}]$, $\mathscr{D}[\mathcal{E}]$ being a linear subspace of $L^{2}$. We put for $\lambda \geqq 0$

$$
\varepsilon_{\lambda}(u, v)=\mathcal{E}(u, v)+\lambda(u, v), u, v \in \mathscr{D}[\mathcal{E}] .
$$

For $\lambda \geqq 0$ and $f \in L^{2}$, denote by $R_{2} f$ a solution $u$ of the next equation:

$$
\left\{\begin{array}{l}
u \in \mathscr{D}[\mathcal{E}] \\
\mathcal{E}_{\lambda}(u, v)=(f, v) \quad v \in \mathscr{D}[\mathcal{E}] .
\end{array}\right.
$$

Our aim is to give an a priori estimate of $R_{\lambda} f$ under the next two conditions on the form $\mathcal{E}$ :

(E.a) If $u \in \mathscr{D}[\mathcal{E}]$ and $k \geqq 0$, then $v=(u-k)^{+} \in \mathscr{D}[\mathcal{E}]$ and $\mathcal{E}(v, v)$ $\leqq \varepsilon(u, v)$.

(E. b) There exist $\lambda_{0} \geqq 0$ and $p_{0}>2$ such that

$$
\|u\|_{p_{0}}^{2} \leqq C \mathcal{E}_{\lambda_{0}}(u, u) \quad u \in \mathscr{D}[\mathcal{E}]
$$

for some constant $C>0$.

Theorem 1. Suppose a bilinear form $\mathcal{E}$ satisfies the conditions (E. a) and (E. b), then for $p>\frac{p_{0}}{p_{0}-2} \vee 2$

$$
\left\|R_{0} f\right\|_{\infty} \leqq C_{1}\|f\|_{p}+C_{2}\left\|R_{0} f\right\|_{2}, \quad f \in L^{p} \cap L^{2}
$$

where $C_{1}(>0)$ and $C_{2}(\geqq 0)$ are constants depending only on $\lambda_{0}, p_{0}, C$

Communicated by K. Itô, October 15, 1976.

* Department of Mathematics, Osaka University, Toyonaka 560, Japan. 
and p. Moreover $C_{2}$ vanishes when $\lambda_{0}=0$ and $m(X)<\infty .{ }^{1)}$

If a bilinear form $\mathcal{E}$ is non-negative definite in the sense that $\mathcal{E}(u$, $u) \geqq 0, u \in \mathscr{D}[\mathcal{E}]$, then the solution $u$ of (1) with $\lambda>0$ is unique and satisfies $\|u\|_{2} \leqq \lambda^{-1}\|f\|_{2}$. Hence we have

Corollary. Suppose a non-negative definite bilinear form $\mathcal{E}$ satisfies ( $\mathcal{E} . \mathrm{a})$ and (E.b), then for $\lambda>0$ and $p>\frac{p_{0}}{p_{0}-2} \vee 2$

$$
\left\|R_{\lambda} f\right\|_{\infty} \leqq C_{1}\|f\|_{p}+C_{2}\|f\|_{2}, \quad f \in L^{p} \cap L^{2}
$$

where $C_{1}(>0)$ and $C_{2}(\geqq 0)$ depend only on $\lambda_{0}, p_{0}, C, \lambda$ and $p$. $C_{2}$ vanishes when $m(X)<\infty$.

G. Stampacchia [7] obtained an estimate very close to (3) when $X$ is a bounded open set $D$ of $R^{n}, m$ is the Lebesgue measure and $\mathcal{E}$ is related to the elliptic differential operator $\sum \frac{\partial}{\partial x_{i}}\left(a_{i j} \frac{\partial}{\partial x_{j}}\right)+\sum b_{i} \frac{\partial}{\partial x_{i}}$ :

$$
\begin{aligned}
\mathcal{E}(u, v)=\sum_{i, j=1}^{n} \int_{D} & \frac{\partial u(x)}{\partial x_{i}} \frac{\partial v(x)}{\partial x_{j}} a_{i j}(x) d x \\
& +\sum_{i=1}^{n} \int_{D} b_{i}(x) \frac{\partial u(x)}{\partial x_{i}} v(x) d x
\end{aligned}
$$

with $\mathscr{D}[\mathcal{E}]=H_{0}{ }^{1}(D)$. Here $a_{i j}$ is bounded, $\sum a_{i j}(x) \xi_{i} \xi_{j} \geqq \nu \mid \xi^{2}, \quad \xi \in R^{n}$, for some constant $\nu>0$ and $b_{i} \in L^{n}(D)$. It is known that this form satisfies the condition ( $\mathcal{E}$. a) even with the equality $\mathcal{E}(v, v)=\mathcal{E}(u, v)$. Moreover property $\left(\mathcal{E}\right.$. b) for a sufficiently large $\lambda_{0}$ (which depends only on $\nu$ and $\left\|b_{i}\right\|_{n}$ ) and for $\frac{1}{2}>\frac{1}{p_{0}}>\frac{1}{2}-\frac{1}{n}$ follows from the coercivity of $\mathcal{E}_{\lambda_{0}}$ on $H_{0}{ }^{1}(D)$ and the Sobolev inequality. ${ }^{2}$

We show in the next section that Stampacchia's method of the proof still works in our general situation of Theorem 1. In Section 3, Theorem 1 and its Corollary are applied to several kinds of Dirichlet forms includ-

1) More explicitly we may take

$$
C_{1}=2^{1+\left(p / p p_{0}-2 p-p_{0}\right)} \cdot C \cdot[m(X)]^{\left(p_{0}-2 / p_{0}\right)-(1 / p)} \text { and } C_{2}=0
$$

when $\lambda_{0}=0$ and $m(X)<\infty$.

2) See footnotes 5 ). 
ing the above one of Stampacchia. In particular we are concerned with a form of non-local character

$$
\mathcal{E}(u, v)=\int_{R^{n}} \int_{R^{n}}(u(x)-u(y))(v(x)-v(y)) k(x, d y) d x
$$

such that $k(x, d y)$ dominates the Lévy measure of the symmetric stable process with index $0<\alpha<2$ :

$$
k(x, E) \geqq \gamma \int_{E}|x-y|^{-(n+\alpha)} d y
$$

for every Borel set $E$ and a.e. $x \in R^{n}, \gamma$ being a positve constant. By making use of a Sobolev type inequality in N. Aronszajn and K. T. Smith $[1]$, we can see that $(\mathcal{E} . \mathrm{b})$ is valid for $\lambda_{0}=1$ and $\frac{1}{2}>\frac{1}{p_{0}}>\frac{1}{2}-\frac{\alpha}{2 n}$.

It has been known ([2] and [3]) that, given a regular Dirichlet form $\mathcal{E}$, there exists uniquely up to a certain equivalence a Hunt process $M$ whose resolvent $R_{\lambda} f$ solves the equation (1). An immediate consequence of our estimate (4) is the absolute continuity of the resolvent kernel $R_{\lambda}$ of the process $\boldsymbol{M}$. Further probabilistic consequences of (4) are stated in the last section. We note here that the estimate like (4) also plays important roles in the study of the stochastic differential equations and stochastic controls ([4]).

\section{§ 2. Proof of Theorem $\mathbb{1}$}

$\left.1^{\circ}\right)$. Given $f \in L^{2} \cap L^{p}$ with $p>2$, consider a solution $u=G_{0} f$ of equation (1) with $\lambda=0$. Put $v=(u-k)^{+}$for a fixed $k>0$, then $v \in \mathscr{D}[\mathcal{E}]$ and $\mathcal{E}(v, v) \leqq(f, v)$ by $(\mathcal{E} . \mathrm{a})$. ( $(\mathcal{E} . \mathrm{b})$ and the Hölder inequality then yield

$$
\|v\|_{p_{0}}^{2} \leqq C(f, v)+C \lambda_{0}[m l(A(k))]^{1-2 / p_{0}}\|v\|_{p_{0}}^{2}
$$

where $A(k)=\{x \in X ; u(x)>k\}$.

Take $k_{0}=\left(2 C \lambda_{0}\right)^{p_{0} / 2 p_{0}-4}\|u\|_{2}$, then the second term of the right hand side of (8) is not greater than $\frac{1}{2}\|v\|_{p_{0}}^{2}$ for $k \geqq k_{0}$, because $m(A(k))$ $\leqq k_{0}{ }^{-2}\|u\|_{2}{ }^{2}=\left(2 C \lambda_{0}\right)^{-p_{0} / p_{0}-2}$ in case that $\lambda_{0}>0$. Hence we get from (8) $\frac{1}{2}\|v\|_{p_{0}}^{2} \leqq C(f, v), k \geqq k_{0}$. Applying the Schwarz inequality to the right hand side and then the Hölder inequality to each factor, we arrive at

$$
\|v\|_{p_{0}} \leqq 2 C\|f\|_{p}[m(A(k))]^{1-1 / p-1 / p_{0}}, \quad k \geqq k_{0} .
$$


It is easy to derive from this for $h>k \geqq k_{0}$

$$
m(A(h)) \leqq \frac{2^{p_{0}} C^{p_{0}}}{(h-k)^{p_{0}}}\|f\|_{p}^{p_{0}}[m(A(k))]^{\left(1-1 / p-1 / p_{0}\right) p_{0}}
$$

$2^{\circ}$ ). From the inequality (9) and Lemma 4.1 of G. Stampacchia [7], we are led to the conclusion that, for $m$-a.e. $x \in X, u(x)$ is dominated by the right hand side of (3) with $C_{2}=\left(2 C \lambda_{0}\right)^{p_{0} / 2 p_{0}-4}$. In case that $\lambda_{0}=0$ and $m(X)=\infty$, we may take $k_{0}=\|u\|_{2}$ and hence $C_{2}=1$ instead of $k_{0}=C_{2}=0$. Applying the same argument as above to $-u$ and $-f$, we can see that $-u(x)$ is also dominated by the same bound $m$-a.e. The proof of Theorem 1 is completed.

\section{§ 3. Application to Dirichlet Forms}

Following H. Kunita [5], we consider in this section a bilinear form $\mathcal{E}$ on $L^{2}$ such that, for some $\mu_{0} \geqq 0, \mathcal{E}_{\mu_{0}}$ is non-negative definite, continuous and closed (see Conditions (B. 1) (B. 3) of [5]). Then there exists uniquely a semigroup $\left\{T_{t}, t>0\right\}$ of operators $T_{t}$ on $L^{2}$ with $\left\|T_{t}\right\|_{2} \leqq e^{\mu_{0} t}$ whose generator $A$ satisfies $\mathcal{E}(u, v)=(-A u, v), u \in \mathscr{D}(A)$, $v \in \mathscr{D}[\mathcal{E}]$. The Laplace transform $R_{d} f$ of $T_{t} f, f \in L^{2}$, is the unique solution of (1) for $\lambda>\mu_{0}$.

Each $T_{t}$ is subMarkov (namely, $0 \leqq T_{t} f \leqq 1$ whenever $0 \leqq f \leqq 1, f$ $\in L^{2}$ ) if and only if $\mathcal{E}$ fulfills the additional condition ( $\mathcal{E}$.a) which is also equivalent to the following [5]:

(E. a') If $u \in \mathscr{D}[\mathcal{E}]$, then $v=(0 \bigvee u) \wedge 1 \in \mathscr{D}[\mathcal{E}]$ and $\mathcal{E}(v, v) \leqq \mathcal{E}(v, u)$. We call $\mathcal{E}$ a Dirichlet form if it satisfies the condition (E.a).

An example of a Dirichlet form for which $\mu_{0}$ is not necessarily zero is the form (5). As was mentioned in Section 1, the form (5) satisfies condition $\left(\mathcal{E}\right.$. a) as well as $\left(\mathcal{E}\right.$. b) (with $\lambda_{0} \geqq \mu_{0}$ ). By Corollary to Theorem 1 we have for $\lambda>\mu_{0}$ and $p>\frac{n}{2} \vee 2$,

$$
\left\|R_{\imath} f\right\|_{\infty} \leqq C_{1}\|f\|_{p}, \quad f \in L^{p}
$$

$C_{1}$ depending only on $\nu,\left\|b_{i}\right\|_{n}, p$ and $\lambda$. The inequality (10) holds even when $0<\lambda \leqq \mu_{0}$ because $R_{\lambda}$ is still bounded on $L^{2}$ ([6; Lemma 2.1]), but it is not clear how the bound is estimated a priori. 
We call a Dirichlet form $\mathcal{E}$ on $L^{2}$ symmetric if $\mu_{0}=0$ and $\mathcal{E}(u, v)$ $=\mathcal{E}(v, u) u, v \in \mathscr{D}[\mathcal{E}]$. A bilinear form $\mathcal{E}$ on $L^{2}$ is a symmetric Dirichlet form if and only if $\mathcal{E}$ is non-negative definite, symmetric, closed and satisfies the condition

(E. a") $u \in \mathscr{D}[\mathcal{E}]$ implies $v=(0 \bigvee u) \wedge 1 \in \mathscr{D}[\mathcal{E}]$ and $\mathcal{E}(v, v) \leqq \mathcal{E}(u, u)$. Symmetric Dirichlet forms on $L^{2}$ are in one-to-one correspondence with semigroups $\left\{T_{\iota}, t>0\right\}$ of strongly continuous symmetric subMarkov operators on $L^{2}$ by the formula

$$
\left\{\begin{array}{l}
\mathscr{D}[\mathcal{E}]=\left\{u \in L^{2} ; \lim _{t \downarrow 0} \frac{1}{t}\left(u-T_{t} u, u\right)<\infty\right\} . \\
\mathcal{E}(u, v)=\lim _{t \downarrow 0} \frac{1}{t}\left(u-T_{\iota} u, v\right) .
\end{array}\right.
$$

Here we give two examples of symmetric Dirichlet forms.

Let $\nu_{i j}, 1 \leqq i, j \leqq n$, be Radon measures on $R^{n}$ such that

$$
\nu_{i j}=\nu_{j i}, \quad \sum_{i, j=1}^{n} \nu_{i j}(E) \xi_{i} \xi_{j} \geqq r \cdot|E| \cdot|\xi|^{2}, \quad \xi \in R^{n}, \quad E \in \mathscr{B}\left(R^{n}\right),
$$

where $\gamma$ is some positive constant and $|E|$ is the Lebesgue measure of $E$. For a fixed open set $D \subset R^{n}$ we put

$$
\mathcal{E}(u, v)=\sum_{i, j=1}^{n} \int_{D}-\frac{\partial u(x)}{\partial x_{i}} \frac{\partial v(x)}{\partial x_{j}} \nu_{i j}(d x), \quad \mathscr{D}[\mathcal{E}]=C_{0}^{\infty}(D) .
$$

We assume that $\mathcal{E}$ is closable on $L^{2}(D)$. Then its smallest closed extension $\bar{\varepsilon}$ is a Dirichlet form on $L^{2}(D)$ ([3]). Furthermore we see from (12) that $\mathscr{D}[\overline{\mathcal{E}}] \subset H_{0}{ }^{1}(D)$ and $r \sum_{i=1}^{n} \int_{D}\left(\frac{\partial u}{\partial x_{i}}\right)^{2} d x \leqq \overline{\mathcal{E}}(u, u), u \in \mathscr{D}[\overline{\mathcal{E}}]$. Hence $\mathcal{E}$ satisfies, in view of the Sobolev inequality, ${ }^{3)}$ the condition ( $\mathcal{E} . \mathrm{b}$ ) with $\frac{1}{2}>\frac{1}{p_{0}}>\frac{1}{2}-\frac{1}{n}$. By Corollary to Theorem 1 , the associated resolvent satisfies (4) for any $\lambda>0$ and $p>\frac{n}{2} \vee 2$, with $C_{1}$ and $C_{2}$ depending only on $\gamma, p$ and $\lambda$. See [3] for examples of $\nu_{i j}$ which are not necessarily absolutely continuous with respect to the Lebesgue measure.

Next let $k(x, E)$ be a kernel on $R^{n} \times \mathscr{B}\left(R^{n}\right)$. We assume the condition (7) as well as the symmetry and finiteness assumptions:

3) See footnotes 5). 


$$
\left\{\begin{array}{l}
\int_{R^{n}} \int_{R^{n}} u(x) v(y) k(x, d y) d x \\
\quad=\int_{R^{n}} \int_{R^{n}} v(x) u(y) k(x, d y) d x, \quad u, v \in C_{0}^{+}\left(R^{n}\right), \\
C_{0}^{\infty}\left(R^{n}\right) \subset \mathscr{D}[\mathcal{E}]
\end{array}\right.
$$

where $\mathcal{E}$ is the form on $L^{2}\left(R^{n}\right)$ defined by (6) with

$$
\mathscr{D}[\mathcal{E}]=\left\{u \in L^{2}\left(R^{n}\right) ; \int_{R^{n}} \int_{R^{n}}(u(x)-u(y))^{2} k(x, d y) d x<\infty\right\} .
$$

It is easy to see that $\mathcal{E}$ is then a symmetric Dirichlet form on $L^{2}\left(R^{n}\right)$.

Denote by $\mathcal{E}^{(\alpha)}$ the symmetric Dirichlet form associated with the convolution semigroup $T_{t} u=\nu_{t}{ }^{*} u$ with $\hat{\nu}_{t}(\xi)=\exp \left(-t|\xi|^{\alpha}\right)$. By the formula (11), we have ${ }^{4)}$

$$
\begin{aligned}
\mathcal{E}^{(\alpha)}(u, u) & =\int_{R^{n}}|\hat{u}(\hat{\xi})|^{2}|\xi|^{\alpha} d \xi \\
& =B(\alpha, n) \int_{R^{n}} \int_{R^{n}}(u(x)-u(y))^{2} \frac{1}{|x-y|^{n+\alpha}} d x d y,
\end{aligned}
$$

where $B(\alpha, n)$ is some universal constant ([1]). $\mathscr{D}\left[\mathcal{E}^{(\alpha)}\right]$ consists of those $u \in L^{2}\left(R^{n}\right)$ for which the integral in (15) converges. Therefore condition (7) leads us to

$$
\mathscr{D}[\mathcal{E}] \subset \mathscr{D}\left[\mathcal{E}^{(\alpha)}\right], \quad \mathcal{E}^{(\alpha)}(u, u) \leqq \frac{B(\alpha, n)}{\gamma} \mathcal{E}(u, u) \quad u \in \mathscr{D}[\mathcal{E}]
$$

Note that the space $\mathscr{D}\left[\mathcal{E}^{(\alpha)}\right]$ and the norm $\sqrt{\mathcal{E}_{1}{ }^{(\alpha)}(u, u)}$ on it coincide respectively with $\boldsymbol{P}_{\alpha / 2}$ and $|u|_{\alpha / 2}$ of N. Aronszajn and K. T. Smith [1]. $\S 10$ of [1] contains a neat proof of the inequality

$$
\|u\|_{p_{0}} \leqq M\|u\|_{\alpha / 2}, \quad u \in \boldsymbol{P}_{\alpha / 2}, \quad \frac{1}{2}>\frac{1}{p_{0}}>\frac{1}{2}--\frac{\alpha}{2 n},
$$

where $M$ is a positive constant and $\|u\|_{\alpha / 2}$ is a certain norm equivalent to $|u|_{\alpha / 2 .}{ }^{5}$ ) Combining this with (16), we get the conclusion stated at

4) $\hat{\nu}_{t}(\xi)=\int_{R^{n}} e^{i \xi \cdot x} u(x) d x$ whereas we put $\hat{u}(\xi)=(2 \pi)^{-n / 2} \int_{R^{n}} e^{i \xi \cdot x} u(x) d x$. Perseval's formula is then in force and $\left(T_{t} u, u\right)=\int \hat{\nu}_{t}(\xi)|\hat{u}(\xi)|^{2} d \xi$.

5) $\|u\|_{a / 2}^{2}=\int_{R^{n}}\left(1+|\xi|^{2}\right)^{a / 2}|\hat{u}(\xi)|^{2} d \xi$. The inequality of Aronszajn-Smith remains valid for $\alpha=2$ in which case the above norm reduces to the norm of the space $H^{1}\left(R^{n}\right)$. The inequality also holds for $\frac{1}{p_{0}}=\frac{1}{2}-\frac{\alpha}{2 n}$ provided $n>\alpha$. 
the end of Section 1. By Corollary to Theorem 1, (4) now holds for $\lambda>0$ and $p>\frac{\alpha}{n} \vee 2$ with $C_{1}$ and $C_{2}$ depending only on $\alpha, \gamma, p$ and $\lambda$.

\section{§4. Probabilistic Consequences}

Let $X$ be a locally compact separable Hausdorff space and $m$ be an everywhere dense positive Radon measure on $X$. If a symmetric Dirichlet form $\mathcal{E}$ on $L^{2}(X ; m)$ is regular ([3]), then there exists an $m$ symmetric standard Markov process $M$ on $X$ whose transition function defines the semigroup on $L^{2}$ associated with $\mathcal{E}$ by the formula (8). $M$ is unique up to an equivalence relative to exceptional sets of zero capacity [3]. Here the capacity of a set is evaluated in terms of $\varepsilon_{1}$. We denote by $p(t, x, E)$ and $R_{\lambda}(x, E)$ the transition function and the resolvent kernel of $\mathbb{M}$ respectively.

Theorem 2. Suppose a symmetric Dirichlet form $\&$ on $L^{2}(X$; $m)$ is regular and satisfies the condition ( $(\mathcal{B} . \mathrm{b})$, then the associated standard process $M$ possesses the following properties: there exists a Borel set $N$ of zero capacity such that $X-N$ is M-invariant and (i) $\quad R_{\lambda}(x, \cdot)$ is absolutely continuous with respect to $m$ for each $\lambda>0$ and $x \in X-N$,

(ii) $p(t, x, \cdot)$ is absolutely continuous with respect to $m$ for each $t>0$ and $x \in X-N$,

(iii) a set $B \subset X-N$ is of zero capacity if and only if $B$ is polar, that is, almost all sample paths starting at $x \in X-N$ do not hit $B$ at positive time.

In fact the assertion (i) follows from the inequality (4) and the quasi-continuity of $R_{\lambda} f, f \in L^{2} \cap C_{0}^{+}$. Properties (i), (ii) and (iii) are equivalent ([3]). If we replace the notion of capacity by the fine capacity, Theorem 2 remains true for any $m$-symmetric standard process whose Dirichlet form satisfies ( $\mathcal{E} . \mathrm{b})$ (without regularity assumption on $\mathcal{E}$ ) ([3]). Theorem 2 can be extended to the non-symmetric Dirichlet form in riew of the work of Carrillo Menender [2]. 


\section{References}

[1] Aronszajn, N. and Smith, K. T., Theory of Bessel potentials, Part I, Ann. Inst. Fourier, Grenoble, 11 (1961), 385-475.

[2] Carrillo Menendez, S., Processus de Markov associé a une forme de Dirichlet non symétrique, Z. Wahrsheinlichkeitstheorie und verw. Gebiete, 33 (1975), 139-154.

[3] Fukushima, M., Potential theory of symmetric Markov processes and its applications, Proc. 3rd Japan-USSR symp. on probability theory, Lecture Notes in Math. 550, Springer-Verlag, 1976.

[4] Krylov, N. V., An inequality on the theory of stochastic integrals, Theory Probability Appl., 16 (1971), 438-448.

[5] Kunita, H., Sub-Markov semi-group in Banach lattice, Proc. International conference on Functional Analysis and related topics, 1969, Tokyo.

[6] Kunita, H., General boundary conditions for multi-dimensional diffusion processes, J. Math. Kyoto Univ., 10 (1970), 273-335.

[7] Stampacchia, G., Le Problème de Dirichlet pour les equations elliptiques du second ordre à coefficent discontinus, Ann. Inst. Fourier, Grenoble, 15 (1965), 189-258. 